\title{
Alteração de deglutição em idosos pós acidente vascular cerebral: Um estudo de revisão integrativa
}

\author{
Alteration of swallowing in the elderly after cerebrovascular accident: An integrative review study \\ Alteración de la deglución en personas mayors después de un accidente cerebrovascular: Un studio \\ de revisión integradora
}

Recebido: 07/07/2021 | Revisado: 15/07/2021 | Aceito: 20/07/2021 | Publicado: 28/07/2021

ORCID: https://orcid.org/0000-0002-0288-0721 Universidade Federal da Paraíba, Brasil E-mail: splfono@msn.com

Ilanna Cibele Delgado de Araújo Fonsêca ORCID: https://orcid.org/0000-0003-1786-7834 Universidade Federal da Paraíba, Brasil E-mail: ilannadelgado@gmail.com

Marcela Leiros Maciel Macêdo ORCID: https://orcid.org/0000-0002-4991-2225 Universidade Federal da Paraíba, Brasil E-mail: marcelaleiros@hotmail.com

Nyellisonn Nando Nóbrega de Lucena ORCID: https://orcid.org/0000-0002-6524-0908 Universidade Federal da Paraíba, Brasil E-mail: nyellisonobrega@hotmail.com Emerson Soares Pontes

ORCID: https://orcid.org/0000-0002-8455-016X Universidade Federal da Paraíba, Brasil E-mail: emerson-soares21@hotmail.com Adriana Queiroga Sarmento ORCID: https://orcid.org/0000-0002-8714-7923 Universidade Federal da Paraíba, Brasil E-mail: aqsguerra@gmail.com

Olívia Galvão Lucena Ferreira

ORCID: https://orcid.org/0000-0002-8490-2444 Universidade Federal da Paraíba, Brasi E-mail: oliviaglf@hotmail.com

Ana Karênina de Freitas Jordão do Amaral ORCID: https://orcid.org/0000-0002-7470-7717 Universidade Federal da Paraíba, Brasil E-mail: akfjafono@hotmail.com

\section{Resumo}

Objetivo:Identificar nas produções científicas as alterações de deglutição em idosos pós-acidente vascular cerebral.Métodos:Revisão integrativa da literatura realizada nas seguintes bases de dados: Lilacs, Scielo, Pubmed Medline e Science Direct. Resultados: Os estudos evidenciaram diversas alterações, categorizadas em alterações alimentares, pulmonares, nutricionais, funcionais e repercussão na qualidade de vida.Conclusão: Ressalta-se a importância de novas pesquisas sobre o tema, evidenciando a importância de um efetivo direcionamento dessas pessoas para uma assistência integral e interdisciplinar, ao profissional de fonoaudiologia e à equipe multidisciplinar, pelos profissionais de saúde.

Palavras-chave: Deglutição; Transtornos de deglutição; Acidente vascular cerebral; Idoso; Fonoaudiologia.

\begin{abstract}
Objective: Identify the alterations of swallowing in the elderly after cerebrovascular accident in scientific productions. Methods: Integrative literature review performed on the following databases: Lilacs, Scielo, Pubmed Medline and Science Direct. Results: The studies evidenced several alterations, categorized as alimentary, pulmonary, nutritional, functional alterations and effects on quality of life. Conclusion: There stands out the importance of new researches on the theme, evidencing the importance of an effective targeting by health professionals of these people to an integral and multidisciplinary assistance, to the speech therapy professional and to the multidisciplinary team.
\end{abstract}

Keywords: Deglutition; Deglutition disorders; Stroke.; Aged; Speech Language and hearing sciences. 


\section{Resumen}

Objective: Identificar, en producciones científicas, alteraciones de la deglución en ancianos tras un ictus.Métodos: Revisión integrativa de la literatura realizada en las siguientes bases de datos: Lilacs, Scielo, Pubmed Medline y Science Direct. Resultados: Los estudios mostraron varios cambios, categorizados en cambios dietéticos, pulmonares, nutricionales, funcionales e impacto en la calidad de vida.Conclusión:Destaca la importancia de profundizar en la investigación sobre el tema, destacando la importancia de dirigir eficazmente a estas personas a una atención integral e interdisciplinar, al patólogo del habla-lenguaje y al equipo multidisciplinario, por parte de los profesionales de la salud.

Palavras-clave: Deglución; Trastornos de la deglución; Accidente vascular cerebral; Ancianos; Terapia del habla.

\section{Introdução}

As repercussões do envelhecimento populacional para a sociedade são consideráveis, especialmente no que diz respeito à saúde. Com o aumento da longevidade, o desafio é viver mais, de forma saudável e com maior qualidade de vida (Rigoto \& Camara, 2016).

$\mathrm{O}$ mundo e o Brasil vêm experimentando um envelhecimento populacional extremamente rápido. $\mathrm{O}$ aumento no número de pessoas idosas ocorre de maneira heterogênea nos diversos países do mundo. No Brasil, em 2010, o IBGE apresentou um aumento na população de idosos de 11\%, havendo um aumento de quase 7 milhões de idosos em 10 anos no país (Costa \& Levefre, 2014) e é possível de se identificar nas previsões populacionais para 2025 com o dobro de pessoas idosas no Brasil e demais países (Brogan \& Blacker, 2014).

As mudanças biopsicossociais no processo do envelhecimento ocorrem de forma heterogênea, tornando essa faixa etária bem peculiar. E o avanço da idade proporciona ao indivíduo mudanças específicas a cada ser humano. Essas mudanças envolvem todo o sistema fisiológico e uma série de mecanismos biológicos como o estilo de vida, a saúde e o estado emocional que resultam numa variabilidade entre as pessoas, na forma e extensão com que as modificações ocorrem(Cardoso, 2012).

O envelhecimento gera a necessidade de um olhar mais dinâmico sobre os idosos e suas consequências naturais, como também sobre as morbidades mais frequentes, principalmente quando o indivíduo não possui uma boa qualidade de vida e o devido cuidado com a sua saúde. Uma patologia cada vez mais frequente é o Acidente Vascular Cerebral (AVC), que se encontra entre os fatores de risco da velhice e representa a primeira causa de morte e incapacidade no país e gera grande impacto econômico e social (Leonardo \& Ferreira, 2013).

O AVC é considerado uma doença crônica não-transmissível (DCNT) que consiste em uma incapacidade neurológica grave não infecciosa, porém limitante, constituindo-se um sério problema de saúde pública. É uma doença cerebrovascular, que representa uma das principais causas de óbitos entre as pessoas com mais de 60 anos e a segunda entre os 15-59 anos (Ribeiro, 2015). O AVC é a terceira causa mais comum de óbito pelas estatísticas divulgadas pela American Heart Association, e é apenas superada pelas doenças cardíacas e cânceres (Sporns \& Eversmann, 2017).

No Brasil, apesar da diminuição nas taxas de mortalidade, o AVC ainda é a principal causa de morte. A população idosa é a que mais se destaca quanto à incidência da doença, que é duplicada após os 60 anos de idade. Considerando ainda a previsão de um crescimento da ordem de trezentos por cento da população idosa nas próximas três décadas, concentrado em países em desenvolvimento, o prognóstico epidemiológico do AVC torna-se ainda mais sombrio (Marchesan \& Santini, 2014).

Além de apresentar uma significativa taxa de letalidade decorrente do AVC, o Brasil apresenta um número expressivo de pessoas com alguma incapacidade física e/ou cognitiva consequência do AVC. Para minimizar os danos dessa fatia da população é necessário o acesso imediato e contínuo aos serviços de reabilitação. Assim, é importante que este acesso ocorra de forma integrada permitindo a pessoa idosa a possibilidade de recuperação (Gatto \& Chelp, 2013).

As alterações ou sequelas do AVC configuram-se como um agravo à saúde, e evidenciam a necessidade do cuidado à pessoa idosa. O seu impacto negativo interfere no exercício da autonomia, na participação social ativa, na capacidade do 
indivíduo em responder às demandas da vida cotidiana, na integração e na qualidade de vida (Sporns \& Eversmann, 2017).

Uma das sequelas mais frequentes que pode ocorrer em decorrência do AVC é o distúrbio de deglutição em pessoas idosas. Aproximadamente dois terços dos pacientes passam a sofrer alterações de deglutição, como consequência de lesões focais decorrentes do AVC. (Jotzz \& Angells, 2017).

A deglutição é vital ao nosso organismo e é resultante de um mecanismo neuromotor, automatizado, cuja função é o transporte do alimento da porção anterior da boca ao estômago. E a alimentação vai além do papel de nutrir e hidratar o indivíduo está associado ao prazer, mas também é um ato necessário à manutenção do nosso corpo em movimento, além da satisfação, à celebração e a coisas boas, bem como, às boas lembranças associadas à alimentação (Jotzz \& Angells, 2017).

$\mathrm{O}$ ato da deglutição, envolve sistemas e estruturas em comum que são: respiratório, circulatório, nervoso, e digestório, assim como, habilidades sensoriais e motoras. Dentre outros fatores, ainda: o nível de consciência e orientação que desempenha um papel importante para a manutenção da socialização, da autonomia e do envelhecimento ativo e saudável (Cassol \& Dassie-Leite, 2012); (Costa \& Levefre, 2014).

A disfagia, constitui-se em um sintoma de dificuldade de deglutição, onde ocorre uma anormalidade no transporte do alimento da boca ao estômago, sendo este processo dividido em algumas etapas sucessivas (Cardoso, 2012). O diagnóstico da alteração de deglutição em pessoas idosas, acometidas por AVC é de extrema relevância. Faz-se necessário que este diagnóstico seja de forma rápida e efetiva, com devido encaminhamento ao profissional de fonoaudiologia, a fim de realizar o acompanhamento pós-hospitalar imediato.

Portanto, estudar as principais alterações de deglutição apresentadas pelos idosos pós AVC torna-se importante para aprofundamento do conhecimento, possibilitando ações e estratégias de cuidado integral e interdisciplinar favorecendo uma reabilitação imediata e prevenção de agravos e complicações, garantindo a promoção da saúde com independência funcional e autonomia (Neumann \& Adami, 2016).

Ao considerar a temática acerca das alterações de deglutição em idosos pós AVC, este estudo busca, responder a seguinte questão norteadora: Quais produções científicas sobre as alterações na deglutição de idosos pós AVC? Portanto, para responder a este questionamento, o estudo tem como objetivo identificar nas produções científicas as alterações de deglutição em idosos pós-acidente vascular cerebral.

\section{Metodologia}

Trata-se de uma revisão integrativa da literatura, que é a mais ampla abordagem metodológica, permitindo a inclusão de estudos experimentais e não-experimentais para uma compreensão completa do fenômeno a ser analisado, seguindo as respectivas etapas: identificação do problema, elaboração da pergunta norteadora, estabelecimento dos critérios de inclusão e exclusão dos artigos, a seleção dos mesmos, e precipuamente a busca de informações relevantes acerca dos artigos revisados (Gaspar \& Leonor, 2015)

Foi realizado um levantamento dos artigos científicos, nas seguintes bases de dados: Lilacs, Scielo, Pubmed, Medline e Science Direct. Para o levantamento dos artigos que fizeram parte da pesquisa foi utilizada a combinação dos seguintes descritores, nas línguas portuguesa, inglesa e espanhola: Acidente Vascular Cerebral, Idoso, Deglutição, Transtornos de Deglutição e Fonoaudiologia, mediados pelo operador booleano AND.

Em seguida, foi realizada leitura dos resumos dos estudos para identificar se estes contemplavam os critérios de inclusão/exclusão estabelecidos e a eliminação das publicações repetidas. Os critérios de inclusão foram: artigos originais publicados em texto completo, em inglês, português e/ou espanhol; publicados nos últimos 10 anos (2008-2017); estudos que abordam a temática de alterações de deglutição em Idosos pós Acidente Vascular Cerebral, nas diversas formas de promoção à saúde, prevenção, avaliação, reabilitação e intervenção. 
Como critérios de exclusão foram definidos: artigos repetidos nas bases de dados, dissertações, teses, cartas ao editor, relatos de caso, relato de experiências; estudos com a faixa etária inferior a 60 anos, estudos em cadáveres, bem como, os que não apresentarem relação com a temática escolhida.

Para a coleta de dados, foi utilizado um roteiro estruturado com as seguintes características: autor (es), ano da publicação, título do artigo, objetivo, periódico, método, tipo de estudo, resultados ou principais achados relacionados as abordagens direcionadas ás alterações de deglutição em Idosos pós Acidente Vascular Cerebral.

Abaixo, a descrição da sequência das buscas nas bases de dados, de acordo com o grupo Prisma:

Figura 1. Sequência de busca nas bases de dados/bibliotecas virtuais de artigos sobre alterações de deglutição em idosos pósAVC, conforme recomendado pelo Grupo PRISMA.

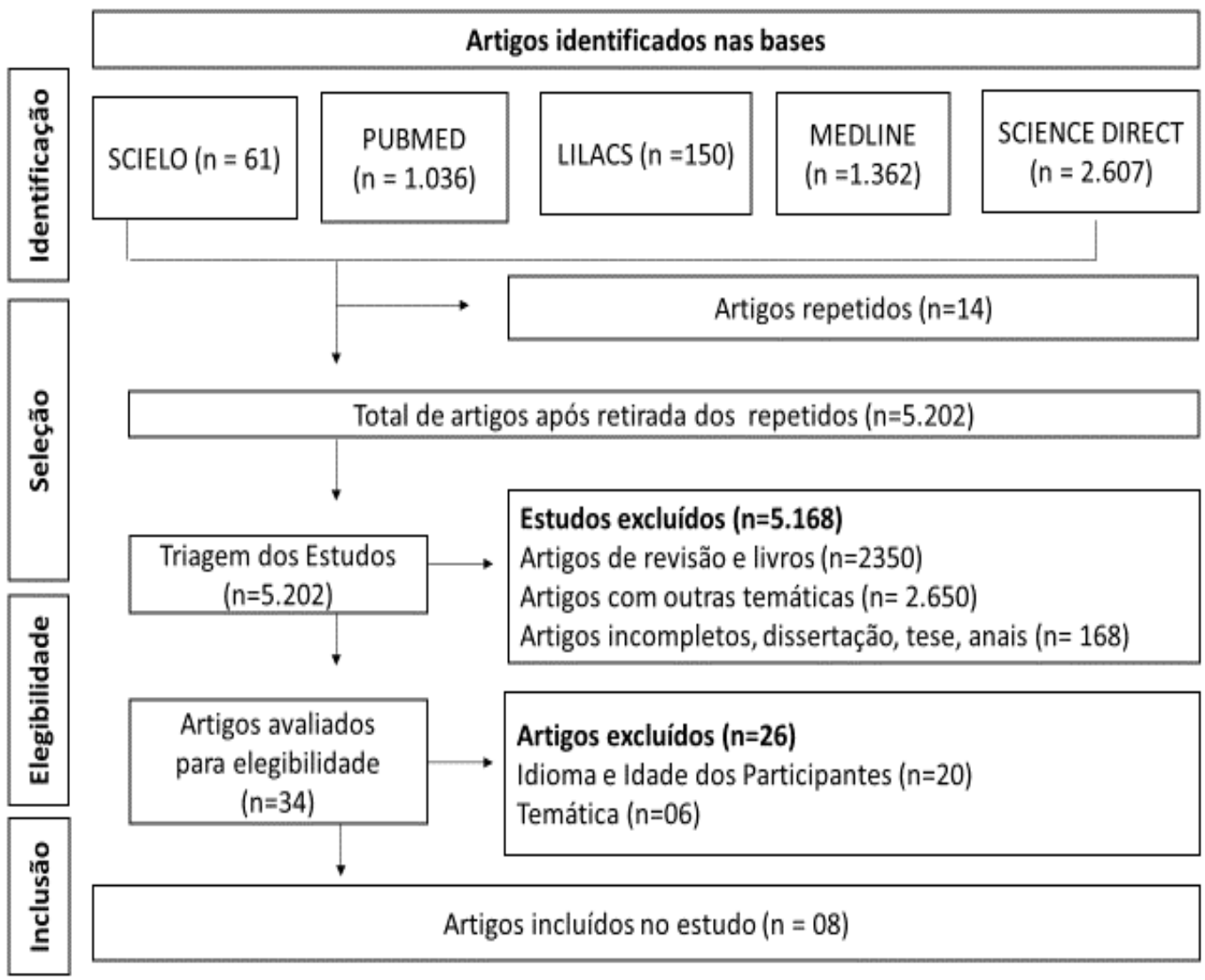

Fonte: Autores

\section{Resultados}

A partir da busca sob os critérios estabelecidos, foram determinados seis artigos que compõem a amostra deste estudo. No Quadro 1, tem-se o resumo das variáveis consideradas. 
Quadro 1. Distribuição dos artigos selecionados, conforme critérios estabelecidos para a pesquisa.

\begin{tabular}{|c|c|c|c|c|}
\hline AUTOR/ANO & TÍTULO & OBJETIVO & $\begin{array}{l}\text { PERIÓDICO/ } \\
\text { E MÉTODO }\end{array}$ & PRINCIPAIS ACHADOS \\
\hline $\begin{array}{c}\text { Brogan E et al., } \\
2014\end{array}$ & $\begin{array}{c}\text { Disfagia e fatores } \\
\text { associados } \\
\text { Infecções Respiratórias na } \\
\text { Primeira Semana } \\
\text { Pós-AVC. }\end{array}$ & $\begin{array}{l}\text { Fornecer evidências sobre } \\
\text { fatores associados a } \\
\text { infecções respiratórias no } \\
\text { pós-AVC. }\end{array}$ & $\begin{array}{l}\text { Neuroepidemiology } \\
\text { Estudo retrospectivo e } \\
\text { de coorte. }\end{array}$ & $\begin{array}{l}\text { A disfagia em idosos no pós- } \\
\text { AVC, causa infecções } \\
\text { respiratórias e consequentemente } \\
\text { aumento no índice de morbidade. }\end{array}$ \\
\hline $\begin{array}{c}\text { Gatto NA et al., } \\
2013\end{array}$ & $\begin{array}{c}\text { Gosto azedo e } \\
\text { temperatura fria na fase } \\
\text { oral da deglutição em } \\
\text { pacientes após acidente } \\
\text { vascular cerebral. }\end{array}$ & $\begin{array}{c}\text { Investigar o efeito do } \\
\text { sabor azedo, e a } \\
\text { temperatura fria, nos } \\
\text { estímulos na fase oral da } \\
\text { deglutição em pacientes } \\
\text { pós AVCI } \\
\end{array}$ & $\begin{array}{c}\text { Codas } \\
\text { Estudo observacional e } \\
\text { transversal. }\end{array}$ & $\begin{array}{l}\text { A disfagia em idosos no pós-AVC } \\
\text { causa uma letargia na contração } \\
\text { de fibras musculares, para efetuar } \\
\text { o processo de deglutição, e assim, } \\
\text { perda de peso acentuada. }\end{array}$ \\
\hline $\begin{array}{c}\text { Paixão CT e } \\
\text { Silva LD, } 2010\end{array}$ & $\begin{array}{l}\text { Características de } \\
\text { pacientes disfágicos em } \\
\text { serviço de atendimento } \\
\text { domiciliar público. }\end{array}$ & $\begin{array}{c}\text { Identificar as } \\
\text { características dos } \\
\text { pacientes portadores de } \\
\text { disfagia decorrentes de } \\
\text { AVC. }\end{array}$ & $\begin{array}{l}\text { Revista Gaúcha de } \\
\text { Enfermagem. } \\
\text { Estudo com abordagem } \\
\text { transversal. }\end{array}$ & $\begin{array}{l}\text { Os transtornos de deglutição em } \\
\text { idosos pós-AVC, causa franqueza } \\
\text { muscular e assim, restrição } \\
\text { quanto à consistência da dieta } \\
\text { alimentar e perda da capacidade } \\
\text { de alimentar-se. }\end{array}$ \\
\hline $\begin{array}{l}\text { Remesso GC et } \\
\text { al., } 2011\end{array}$ & $\begin{array}{l}\text { Alteração da deglutição } \\
\text { após AVCI. }\end{array}$ & $\begin{array}{l}\text { Investigaram ocorrência } \\
\text { de distúrbios da } \\
\text { deglutição após AVC. }\end{array}$ & $\begin{array}{l}\text { Arq. Neuropsiquiatria. } \\
\text { Abordagem transversal } \\
\text { e observacional }\end{array}$ & $\begin{array}{l}\text { Os distúrbios da deglutição em } \\
\text { idosos pós-AVC, causa } \\
\text { dificuldade respiratória e } \\
\text { consequentemente infecções } \\
\text { respiratórias. } \\
\end{array}$ \\
\hline $\begin{array}{l}\text { Ribeiro PW, } \\
2015\end{array}$ & $\begin{array}{c}\text { Relação entre Disfagia, } \\
\text { Institutos Nacionais de } \\
\text { Escore de Escala de AVC } \\
\text { e Preditores de } \\
\text { Pneumonia após AVCI }\end{array}$ & $\begin{array}{c}\text { Avaliar a relação entre o } \\
\text { escore do NIHSS e a } \\
\text { presença de penetração e } \\
\text { laríngea e/ou } \\
\text { laringotraqueal e } \\
\text { aspiração; identificar } \\
\text { quais fatores são } \\
\text { preditores da ocorrência } \\
\text { de pneumonia. } \\
\end{array}$ & $\begin{array}{c}\text { Journal of Stroke and } \\
\text { Cerebrovascular } \\
\text { Diseases. } \\
\text { Estudo observacional, } \\
\text { transversal e } \\
\text { prospectivo }\end{array}$ & $\begin{array}{l}\text { Idosos pós-AVCI, hospitalizados } \\
\text { e disfágicos, apresentaram déficit } \\
\text { no reflexo de tosse e maior } \\
\text { probabilidade de desenvolver } \\
\text { pneumonia. }\end{array}$ \\
\hline $\begin{array}{c}\text { Sporns PB et al, } \\
2017\end{array}$ & $\begin{array}{l}\text { A atrofia dos músculos da } \\
\text { deglutição está associada } \\
\text { à gravidade da } \\
\text { disfagia e idade em } \\
\text { Pacientes com Acidente } \\
\text { Vascular Cerebral. }\end{array}$ & $\begin{array}{c}\text { Avaliar se existe } \\
\text { correlação entre idade e } \\
\text { volume muscular, estando } \\
\text { relacionado à } \\
\text { disfagia no pós-AVCI. }\end{array}$ & $\begin{array}{c}\text { Journal JAMDA } \\
\text { Estudo retrospectivo }\end{array}$ & $\begin{array}{l}\text { A disfagia em idosos decorrentes } \\
\text { de AVC causa atrofia muscular } \\
\text { (sarcopenia) gerando, aumento no } \\
\text { tempo de alimentação e ainda } \\
\text { modificação na consistência } \\
\text { alimentar, e consequentemente a } \\
\text { colocação de uma via alternativa } \\
\text { de alimentação. }\end{array}$ \\
\hline
\end{tabular}

Fonte: Autores.

\section{Discussão}

A partir da análise dos achados, emergiram quatro categorias temáticas: 1. Alterações Alimentares; 2. Alterações Pulmonares e Nutricionais; 3. Alterações Funcionais; 4. Repercussão na qualidade de vida, que serão discutidas a seguir.

\section{Categoria 1: Alterações Alimentares}

Uma grande parte dos indivíduos idosos acometidos por AVC, tem como consequência algumas incapacidades, sendo a mais encontrada a fraqueza muscular e conseguinte a perda da capacidade de se alimentar, com as mudanças ou adaptações nas consistências alimentares e assim, os transtornos de deglutição. Essa incapacidade gera uma restrição que resulta numa deficiência ou falta de habilidade para desempenhar uma atividade normal para os seres humanos (Souza \& Pereira, 2011).

A disfagia em idosos, acometidos por $\mathrm{AVC}$, tem como consequência algumas incapacidades, sendo as mais encontradas as alterações nos aspectos alimentares, com mudanças ou adaptações nas consistências alimentares e consequentemente, surgimento dos transtornos de deglutição, que podem acarretar na introdução de uma via alternativa de 
alimentação como: a sonda nasoenteral, sonda nasogástrica, a gastrostomia e a sonda jejunal, deixando de alimentar-se por via oral (Souza \& Pereira, 2011).

Em geral, pacientes com sequelas de AVC, que envolvam danos ao hemisfério direito tendem a apresentar problemas na deglutição, dessa forma, a localização do AVC é o fator mais determinante do que, o número de episódios de AVC para o acometimento do distúrbio de deglutição (Paixão \& Silva, 2010).

\section{Categoria 2: Alterações Pulmonares e Nutricionais}

Os distúrbios da deglutição após AVC são ocorrências comuns, com alta incidência e complicações, ao qual evidencia o aumento da mortalidade, devido aos riscos pulmonares por aspiração, desidratação, desnutrição e períodos de internação hospitalar prolongado (Remesso \& Prado, 2011).

Idosos com sequela de AVC que apresentam alterações de deglutição, consequentemente acarretam em prejuízos nos aspectos nutricionais, de hidratação e nos aspectos pulmonares no estado pulmonar, e assim, trazendo riscos de desnutrição, pneumonia por broncoaspiração e desidratação (Souza \& Pereira, 2011).

Algumas das questões evidenciadas na disfagia em idosos acometidos pelo AVC, é a condição do padrão respiratório que comumente encontra-se alterado, e por consequência a pneumonias consecutivas, elevando os índices de morbidade em idosos, e ainda a perda de peso acentuada levando a quadro de desnutrição e desidratação, levando a implicações de ordem biológicas, mas também psicológica e social. Assim, tal morbidade pode ocasionar prejuízos nos aspectos nutricionais, de hidratação e pulmonar, podendo levar a perda descontrolável de peso ou desnutrição e até mesmo a morte (Costa \& Levefre, 2014).

\section{Categoria 3: Alterações Funcionais}

A disfagia, aparece como uma incapacidade que contribui para a perda da funcionalidade e independência, bem como, nos hábitos funcionais, como: a perda da força muscular e a diminuição da sensibilidade oral, em idosos pós-AVC devido as alterações vivenciadas no momento da alimentação e deglutição, são consideráveis (Oms, 2015). Sabendo que a composição muscular se modifica com a idade aumentada, e os componentes e a coordenação entre eles diminuem, com a diminuição do número de fibras musculares e a redução de enzimas que retardam a contração muscular, de forma fisiológica, as dificuldades relacionadas à deglutição podem assim ser justificadas (Acosta \& Cardoso, 2013).

Essas características causam alterações funcionais na deglutição em idosos no pós AVC, como pode-se destacar: restos alimentares após a ingestão alimentar em cavidade oral, reflexo palatino anormal, dificuldade na mastigação, atraso na fase oral e faríngea, que culminam nos achados encontrados nos transtornos de deglutição propriamente ditos, como: a qualidade vocal molhada, tosse fraca ou ausente, a tosse voluntária ausente, aumento da secreção e de alteração em seu controle; episódios de tosse, pigarro e engasgos, após a deglutição, penetração laríngea, podendo evoluir para uma aspiração laringotraqueal (Remesso \& Prado, 2011).

Além dessa condição, a diminuição da quantidade de saliva contribui na diminuição da força e mobilidade das estruturas fonoarticulatórias, e consequentemente dificultando a atuação do sistema estomatognático, são agravados pelo AVC (Gaspar \& Leonor, 2015). E acarreta prejuízos no prazer em se alimentar influenciando no desejo ou vontade de comer, na mudança da dieta alimentar quanto à consistência preferindo alimentos mais amolecidos, apresentam cansaço ou fadiga durante a ingestão alimentar, déficit do reflexo de tosse e consequente probabilidade de desenvolver pneumonia. E assim desenvolvendo riscos à saúde da pessoa idosa e em seu equilíbrio social (Brogan \& Blacker, 2014).

A responsabilidade para o diagnóstico e tratamento de idosos acometidos por AVC, e que apresentam distúrbios de deglutição, a fim de que alcancem assistência ampla e integral, é necessária junto à equipe multidisciplinar. Dessa forma é 
possível garantir condições de acesso a estes profissionais, bem como, o encaminhamento ao profissional de fonoaudiologia e a equipe multidisciplinar (Gaspar \& Leonor, 2015).

\section{Categoria 4: Repercussão na qualidade de vida}

Conceituar a qualidade de vida é algo subjetivo, multidimensional, sofre influências de vários aspectos relacionados à educação, economia e aspectos socioculturais, como a satisfação pessoal do indivíduo em relação à expectativa de vida, percepção da saúde, interesses, aspectos físicos, emocionais e socioeconômicos (Winstein, 2016).

As restrições de deglutição vivenciadas pelos idosos podem trazer alguns sentimentos de desânimo, frustração, vergonha, constrangimento diante dos familiares e amigos, e esses sentimentos podem levá-los ao isolamento. Podem passar a realizar, cada vez mais, as refeições sozinhas ou evitar se alimentar na frente de outros, resultando no isolamento social e aumentando o risco consequente de uma depressão, considerando que a alimentação, traz em sua essência um momento de interação social e de agradável convívio interpessoal ${ }^{10}$

O real impacto que as alterações de deglutição causam na qualidade de vida dos idosos pós AVC, são consideráveis devido Às alterações vivenciadas no momento da alimentação e da deglutição, que não apenas representa o papel nutricional e de hidratação e sim o papel social e de comunicação entre os indivíduos, assim gerando isolamento social e acarretando em depressão (Cunha, 2016); (Chaves, 2019).

Entretanto, se faz necessário avaliar o que o distúrbio de deglutição acarreta na qualidade de vida da pessoa idosa, a fim de se conhecer quais os verdadeiros impactos das alterações vivenciadas no momento da alimentação. Em estudo refere que idosos sem disfagia, apresentam dor, porém melhor estado geral de saúde, e vitalidade do que pessoas idosas com disfagia, bem como, a capacidade funcional melhor neste grupo ${ }^{4}$. Poucos estudos estão disponíveis sobre o impacto da disfagia na qualidade de vida do idoso. A disfagia pode ocorrer após o AVC em idosos e os primeiros sintomas dependem da localização e extensão da lesão neurológica (Gatto \& Chelp, 2013).

Estudos acerca do impacto das alterações da deglutição na qualidade de vida das pessoas idosas pós AVC, ainda são escassos, entretanto, se faz necessário novos estudos sobre essa temática a fim de se conhecer as repercussões na qualidade de vida e as estratégias de enfrentamento para uma assistência à saúde interdisciplinar que alcance as necessidades não apenas biológicas mas considere aspectos psicológicos e sociais (Marchesan, 2014); ( Cunha, 2016); (Chaves, 2019).

\section{Conclusão}

As produções científicas analisadas referiam acerca das alterações de deglutição que a pessoa idosa apresenta após o AVC e entre elas destacam-se: diminuição na vontade e no prazer em alimentar-se, fadiga e cansaço durante a alimentação, mudança de consistência alimentar, perda da capacidade de alimentar-se, com consequente colocação de via alternativa de alimentação, déficit no reflexo de tosse e na sensibilidade intra-oral, riscos de infecções respiratórias como pneumonias e aumento de morbidade, e ainda na qualidade de vida. As alterações evidenciadas nos estudos dessa amostra foram categorizadas em alterações alimentares, pulmonares, nutricionais, funcionais e repercussão na qualidade de vida.

$\mathrm{O}$ acidente vascular cerebral é um dos cinco descritores mais citados nas pesquisas atuais, porém as alterações de deglutição em idosos torna-se restrito. Isto é algo preocupante, para manter ativa e saudável a vida das pessoas mais velhas, pois uma meta fundamental é garantir a autonomia e a independência no processo de envelhecimento, ressaltando que a alimentação é um forte aliado da vida social e ela precisa ser realizada com segurança.

Ressalta-se a importância de novas pesquisas sobre o tema, tendo em vista que os achados encontrados são diversos, assim como, estudos que evidencie a importância de um efetivo direcionamento dessas pessoas para uma assistência integral e 
interdisciplinar, ao profissional de fonoaudiologia e a equipe multidisciplinar, pelos profissionais de saúde.

Como sugestão para futuras pesquisas, sugere-se que as alterações de deglutição em idosos pós acidente vascular cerebral, amplie seu espectro de pesquisa na área e que haja divulgação para diversas categorias profissionais de saúde, auxiliando no cuidado ao idoso disfágico.

\section{Referências}

Acosta, N.(2013). Presbifagia: estado da arte da deglutição do idoso. RBCEH, 9(1), 143-154. São Paulo http://seer.upf.br/index.php/rbceh/article/view/1504/

Brogan, E. (2014). Dysphagia and Factors Associated with Respiratory Infections in the First Week Post Stroke. Neuroepidemiology,43(2), 140-144. Porto Alegre https://www.karger.com/Article/Abstract/366423.

Cardoso, M. (2012). Disfagias Orofaríngeas: implicações clínicas. Roca

Cassol, K. (2012). Qualidade de vida em deglutição em idosos saudáveis. J. Soc. Bras. Fonoaudiol, 24(3), 223-232. São Paulo http://www.scielo.br/scielo. php?Script=sci_arttext\&pid=S2179-64912012000300007\&lng=en. http://dx.doi.org/10. 1590/S2179-64912012000300007/

Chaves, S.(2019). Disfagia em idosos pós AVC: checklist para rastreamento. Rev. Riase,5(2),377-390.Portugal http://www.revistas.uevora.pt/ index.php/saude_en velhecimento/article/view/

Costa, M.(2014). Doenças crônicas não transmissíveis - cuidado em fonoaudiologia. Guanabara

Cunha, D.(2016). Contribuição fonoaudiológica em idosos acometidos de acidente vascular Encefálico. Rev. bras. geriatr. gerontol., 10(3), 25-28. https://pesquisa.bvsalud.org/portal/resource/pt/biblio-905351/

Gaspar, M.(2015). Avaliação da qualidade de vida em pacientes com disfagia neurogênica. Rev. CEFAC, 17(6), 1939-1945. http://www.scielo.br/scielo. php?script=sci arttext\&pid=S1516-18462015000801939\&lng=en. http://dx.doi.org/10.1590/1982-0216201517619114/

Gatto, N.(2013). Sour taste and cold temperature in the oral phase of swallowing in patients after stroke. CODAS, 25(2), 163-167. http://www.scielo.br/scielo. Php?script=sci_arttext\&pid=S231717822013000200012\&lng=en. http://dx.doi.org/10.1590/S2317-17822013000200012

Leonard, M(2013). O AVC no processo do envelhecimento: uma avaliação da capacidade funcional em estudo de caso-controle. Campina Grande: Cieh

Marchesan, I.(2014). Deglutição: normalidade. Disfagias orofaríngeas. Pró-fono

Ribeiro, P.(2015).W. Relationship between dysphagia, national institutes of health stroke scale score, and predictors of pneumonia after ischemic stroke. $J$. Stroke Cerebrovasc. Diseases, 24(9), 2088-2094. https://www.ncbi.nlm.nih.gov/pubmed/26187787. 10.1016/j.jstrokecerebrovasdis. 2015.05.009.21

Rigoto, M.(2016). Contribuição fonoaudiológica em idosos pós-acidente vascular cerebral.Rev.bras.geriatr.gerontol., 2(10),25-28. http://www.cuidadoéfundamental.br/

Sporns, P.(2017). Atrophy of swallowing muscles is associated with severity of dysphagia and age in patients with acute stroke. J. Am. Med. Dir. Assoc.,18(7), 635-650. Estados Unidos https://www.ncbi.nlm.nih.gov/pubmed/28363443/

Jotz, G.(2017). Disfagia: abordagem clínica e cirúrgica: criança, adulto e idoso. Elsevier.

Neumann, L.(2016). Sensibilidade gustativa de adultos e idosos. Rev bras geriatr. gerontol., 9(5), 797-808. http://www.scielo.br/pdf/rbgg/v19n5/pt_18099823-rbgg-19-05-00797/

Organização Mundial da Saúde. (2015). Relatório mundial de envelhecimento e saúde. 2 de agosto, 2018, Genebra http://sbgg.org.br/wp-content/uploads/

Paixão, C.(2010). Características de pacientes disfágicos em serviço de atendimento domiciliar público. Rev. gaúcha enferm, 31(2), 262-269. http://www.scielo.br/scielo. php?script=sci_arttext\&pid=S1983-14472010000200009\&lng=en. http://dx.doi.org/10. 1590/S1983-14472010000200009.

Remesso, G.(2011). Alterações da deglutição após acidente cerebrovascular isquêmico. Arq. Neuro-Psiquiatr.,69(5), 785-789. https://www.scielo.br/j/anp/a/ykcdk58rRW67ckzzbzLQHrS/?lang=en/

Souza, A. (2011). O grupo na fonoaudiologia: origens clínicas e na Saúde Coletiva. Rev CEFAC. 13(1), 140-51. http://www.scielo.br/scielo.php?Script=sci_arttext\&pid=S1516-18462011000100017\&lng=en. http://dx.doi.org/10.1590/S1516-18462010005000042/

Winstein, C.(2016). Guidelines for adult stroke rehabilitation and recovery: a guideline for healthcare professionals from the American Heart Association.American Stroke Association, 47(1), 1224-1236. Estados Unidos https://www.ahajournals.org/doi/full/10.1161/STR.0000000000000098/ 